\title{
Quark Mass Dependence of the QCD Critical End Point in the Strong Coupling Limit
}

\author{
Jangho Kim* \\ Fakultät für Physik, Universität Bielefeld, Universitätstasse 25, D33619 Bielefeld, Germany \\ E-mail: jangho@physik.uni-bielfeld.de \\ Wolfgang Unger \\ Fakultät für Physik, Universität Bielefeld, Universitätstasse 25, D33619 Bielefeld, Germany \\ E-mail: wunger@physik.uni-bielfeld.de
}

Strong coupling lattice QCD in the dual representation allows to study the full $\mu-T$ phase diagram, due to the mildness of the finite density sign problem. Such simulations have been performed in the chiral limit, both at finite $N_{t}$ and in the continuous time limit. Here we extend the phase diagram to finite quark masses, with an emphasis on the low temperature first order transition. We present our results on the quark mass dependence of the critical end point and the first order line obtained by Monte Carlo via the worm algorithm.

34th annual International Symposium on Lattice Field Theory

24-30 July 2016

University of Southampton, UK

*Speaker. 


\section{Introduction}

It is possible to investigate the full $\mu-T$ phase diagram using strong coupling lattice QCD in the dual representation due to its mild sign problem. The sign problem depends on the representation of the partition function. It is well known that in the strong coupling limit $\beta \equiv \frac{2 N_{c}}{g^{2}} \rightarrow 0$, i. e. in the absence of the gauge action, one can make use of a dual representation due to the factorization of one-link gauge integrals [1]. This dual representation is well suited for Monte Carlo simulations via the worm algorithm $[2,3]$. Such simulations on the $\mu-T$ phase diagram have been carried out in the chiral limit [4,5]. Simulation at finite quark masses have been studied in [3]. Here we extend on these studies and focus on the phase boundary for finite quark masses. Also we obtain the critical end points (CEP) for finite quark masses.

The Lagrangian for staggered fermions $\chi$ including an anisotropy $\gamma$, favoring temporal gauge links in order to continuously vary the temperature, is:

$$
\mathscr{L}_{F}=\sum_{v} \frac{\gamma^{\delta_{v 0}}}{2} \eta_{v}(x)\left(e^{\mu \delta_{v 0}} \bar{\chi}(x) U_{v}(x) \chi(x+\hat{v})-e^{-\mu \delta_{v 0}} \bar{\chi}(x+\hat{v}) U_{v}^{\dagger}(x) \chi(x)\right) .
$$

From the Eq. (1.1), one can derive the partition function in the dual representation by integrating out the gauge links and Grassmann variables:

$$
Z=\sum_{\{k, n, \ell\}} \underbrace{\prod_{b=(x, \hat{\mu})} \frac{\left(N_{c}-k_{b}\right) !}{N_{c} ! k_{b} !} \gamma^{2 k_{b} \delta_{\hat{0}, \hat{\mu}}}}_{\text {meson hoppings }} \underbrace{\prod_{x} \frac{N_{c} !}{n_{x} !}\left(2 a m_{q}\right)^{n_{x}}}_{\text {chiral condensate }} \underbrace{\prod_{\ell} w(\ell, \mu)}_{\text {baryon hoppings }}
$$

This partition function describes a system of mesons and baryons. The mesons live on the bonds $b \equiv(x, \hat{\mu})$, where they hop to a nearest neighbor $y=x+\hat{\mu}$, and the hopping multiplicity are given by so-called dimers $k_{b} \in\left\{0, \ldots, N_{c}\right\}$. The baryon must form self-avoiding loops.

$$
w(\ell, \mu)=\frac{1}{\prod_{x \in \ell}} \sigma(\ell) \gamma^{3 N_{\hat{0}}} \exp \left(3 N_{t} r_{\ell} a_{t} \mu\right), \quad \sigma(\ell)=(-1)^{r_{\ell}+N_{-}(\ell)+1} \prod_{b=(x, \hat{\mu}) \in \ell} \eta_{\hat{\mu}}(x),
$$

where $\ell$ denotes a baryon loop, $N_{\hat{0}}$ is the number of baryons in temporal direction. $N_{t}$ is the number of lattice sites in temporal direction and $r_{\ell}$ is the baryon winding number in temporal direction. $\sigma(\ell)$ is the sign. The sign is related to staggered phase factor $\eta_{\hat{\mu}}(x)$ and the geometry of the baryon loop $\ell$ : the winding number $r_{\ell}$ and the number of baryons in negative direction $N_{-}(\ell) . N_{-}(\ell)$ comes from the negative sign in front of the second term in Eq. (1.1). By the Grassmann constraint, the summation over configurations $\sum_{\{k, n, \ell\}}$ in Eq. (1.1) is restricted by the following condition.

$$
n_{x}+\sum_{\mu= \pm 0, \cdots, \pm d}\left(k_{\mu}(x)+\frac{N_{c}}{2}\left|\ell_{\mu}(x)\right|\right)=N_{c}
$$

In the chiral condensate part, $m_{q}$ is the quark mass and $n_{x}$ is the number of monomers at site $x$. In the chiral limit, monomers are absent to avoid that the partition function becomes zero. On the contrary, for finite quark masses, monomers are present. 


\section{Chiral and Nuclear Transition}

\subsection{Symmetries and phase diagram}

The chiral symmetry at strong coupling is $\chi^{\prime}(x)=e^{i \alpha \varepsilon(x)} \chi(x)$, where $\varepsilon(x)=(-1)^{\sum_{\mu} x_{\mu}}$. It is spontaneously broken $(\langle\bar{\chi} \chi\rangle \neq 0)$ at low temperatures and densities. At high temperatures and densities, the chiral symmetry is restored $(\langle\bar{\chi} \chi\rangle=0)$. Between these two phases, there is a 2nd order phase transition line with $O(2)$ critical exponents at small chemical potential $\left(\mu<\mu_{\text {tric }}\right)$ and a 1 st order line $\mu_{1 \mathrm{st}}(T)>\mu_{\text {tric }}$. The tricritical point (TCP) is located between the 2 nd and 1 st order lines point. On the other hand, nuclear transition between vacuum phase and nuclear matter phase does not have the 2 nd order line. They have the 2 nd order CEP that is similar to the CEP of a liquid gas transition, and the 1 st order line is located below the CEP. The 1 st order line $\mu_{1 \mathrm{st}}(T)$ separates the hadronic phase where the baryon density $\left\langle n_{B}\right\rangle=0$ from the nuclear matter phase. At $T=0$ and above $\mu>\mu_{1 \text { st }}$, where $\left\langle n_{B}\right\rangle=1$, Pauli saturation occurrs: Due to the finite lattice spacing, the baryons form a crystal in this nuclear matter phase, i. e. every lattice site is filled by a baryon. Because of the Pauli principle, the mesons and the baryons can not intersect with each other. Hence, in the nuclear matter phase, the chiral condensate $\langle\bar{\chi} \chi\rangle$ vanishes. On the contrary, in the hadronic phase, the baryons are rare and the mesons are common.

\subsection{Observables}

Our observables for the chiral transition are the chiral condensate $\langle\bar{\chi} \chi\rangle$ and the chiral susceptibility $\chi_{c h}$.

$$
\langle\bar{\chi} \chi\rangle=\frac{1}{2 m_{q} V}\left\langle N_{M}\right\rangle, \quad \chi_{c h}=\frac{1}{V} \frac{\partial^{2}}{\partial\left(2 m_{q}\right)^{2}} \log Z=\frac{1}{\left(2 m_{q}\right)^{2} V}\left(\left\langle N_{M}^{2}\right\rangle-\left\langle N_{M}\right\rangle^{2}-\left\langle N_{M}\right\rangle\right),
$$

where $N_{M} \equiv \sum_{x} n_{x}$. In the chiral limit, $\langle\bar{\chi} \chi\rangle=0$ because $N_{M}=0$. For the nuclear transition, we measure the baryon density $\left\langle n_{B}\right\rangle$ and the baryon susceptibility $\chi_{B}$, which are given by the winding numbers $r_{\ell} \in \mathbb{Z}$.

$$
\left\langle n_{B}\right\rangle=\frac{1}{V_{s} N_{t}} \frac{\partial}{\partial\left(3 a_{t} \mu\right)} \log Z=\frac{1}{V_{s}}\left\langle\sum_{\ell} r_{\ell}\right\rangle, \quad \chi_{B}=\frac{N_{t}}{V_{s}}\left(\left\langle\left(\sum_{\ell} r_{\ell}\right)^{2}\right\rangle-\left\langle\sum_{\ell} r_{\ell}\right\rangle^{2}\right)
$$

The general reweighting method with the average sign is applied in our study.

$$
\langle O\rangle=\frac{\langle O \sigma\rangle}{\langle\sigma\rangle}, \quad\langle\sigma\rangle=\exp \left(-L^{3} N_{t} \Delta f\right),
$$

where $\Delta f$ is the difference between full and sign-quenched free energy density.

\subsection{Anisotropy and finite temperature}

We introduce the anisotropy $\gamma$ in the Dirac couplings in order to vary the temperature in the strong coupling limit $\beta=0$, where $a$ does not vary, but $a_{t}$ does vary in Eq. (1.1). The ratio of the lattice spacing in spatial and temporal direction can be written as a general function $\frac{a}{a_{t}}=\xi(\gamma)$. Mean field theory of Eq. (1.2) suggests [6] that $\xi^{\prime}(\gamma)=\gamma^{2}$, it is an $N_{t}$-independent choice. $\xi(\gamma)$ is obtained from non-perturbative calculation [7]. Hence, we use the following notations to distinguish $\xi(\gamma)$ and $\xi^{\prime}(\gamma)$.

$$
a \mu^{\prime}=a_{t} \mu \xi^{\prime}(\gamma), \quad a T^{\prime}=\frac{\xi^{\prime}(\gamma)}{N_{t}}, \quad a \mu=a_{t} \mu \xi(\gamma), \quad a T=\frac{\xi(\gamma)}{N_{t}}
$$




\section{Results}

\subsection{Average sign}
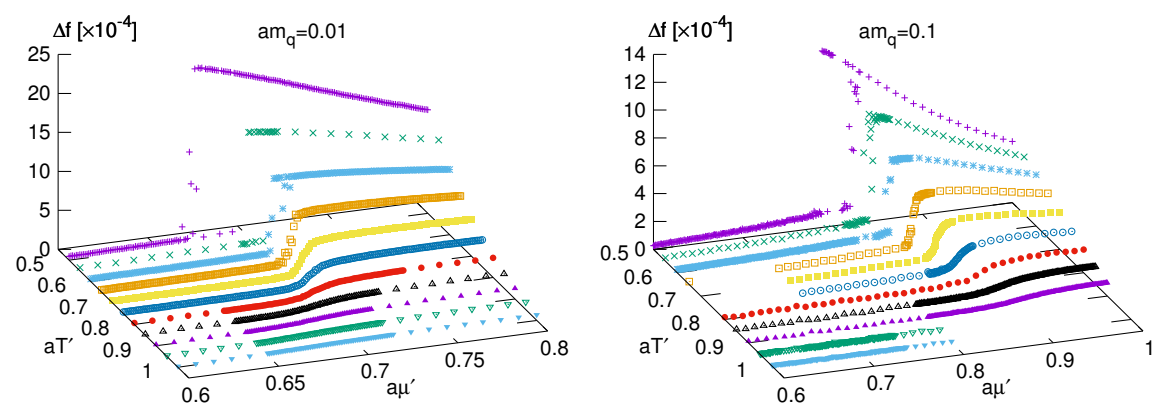

Figure 1: The average sign in the range of $0.5<a T^{\prime}<1$, and $0.6<a \mu^{\prime}<0.8$ for $a m_{q}=0.01,0.1$. Here, $\Delta f=-\frac{1}{V} \log \langle\operatorname{sign}\rangle$

In order to show the mildness of the sign problem in the dual the representation, we plot $\Delta f$ in Fig. 1 which is defined in Eq. (2.3). In the area of $0.5<a T^{\prime}<1$, and $0.6<a \mu^{\prime}<0.8$, where our simulation is done, the average sign is quite small for various quark masses $a m_{q}=0.01,0.1$.

\subsection{Finite size scaling at finite density}

We use finite size scaling to the chiral and nuclear susceptibilities to find the temperature of CEP. The finite size scaling is carried out using the following critical exponents. In the chiral limit $\left(m_{q}=0\right)$, the $O(2)$ exponents with $L^{\rho(\equiv / v)}, \gamma=1.3177$ and $v=0.67155$ are used for the 2nd order line in chiral transition and CEP in nuclear transition. For the crossover region in the nuclear transition, $\rho=1$ is applied. We use the exponents with $\gamma=1$ and $v=0.5$ at the TCP for the chiral transition. For the finite quark masses, we apply the $Z(2)$ exponents with $\gamma=1.237$ and $v=0.613$ at the CEP for both chiral and nuclear transitions. $\rho=3$ is applied for the first order lines.

We scan the parameter space along the $\mu$-direction for various temperatures in the range of $0.5 \leq a T \leq 1.0$ with the step size 0.05 to find the CEP and phase boundary. We analyse a peak of the chiral and baryon susceptibilities. In the chiral limit, the chiral susceptibility does not have a peak. Hence, we use the baryon susceptibility to find the TCP temperature because the location of the TCP in the chiral transition is same as that of the CEP for the nuclear transition in the strong coupling limit $(\beta=0)$. For finite quark masses, the chiral susceptibility has a peak. So, we obtain the CEPs and phase boundaries separately from the chiral and nuclear transitions. We use the standard finite scaling method to find the temperature of the CEP. We compare the peak heights of the different lattice volumes, they are rescaled by the CEP exponents. By the standard method, the peak heights of the different lattice volumes become equal at the temperature $\left(a T_{E}^{\prime}\right)$ of the CEP.

To obtain the critical chemical potential $a_{t} \mu_{c}^{\prime}$, we analyse the peak position of the chiral and baryon susceptibilities. For the chiral transition in the chiral limit, we obtain the critical chemical potential $a_{t} \mu_{c}^{\prime}$ from the crossing points between the different lattice volumes because the chiral susceptibility does not have a peak. For other cases, we obtain the peak position using the following 


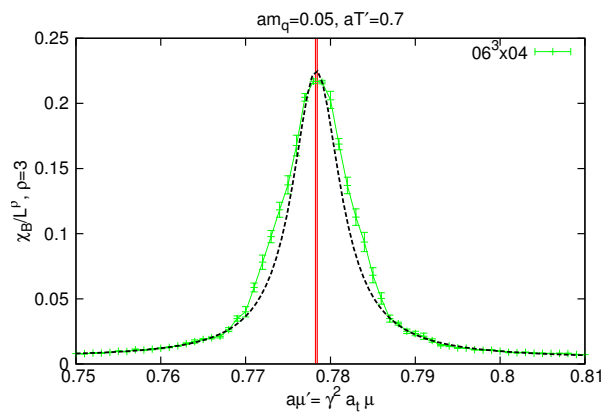

(a) Fit to the Breit-Wigner with polynomial

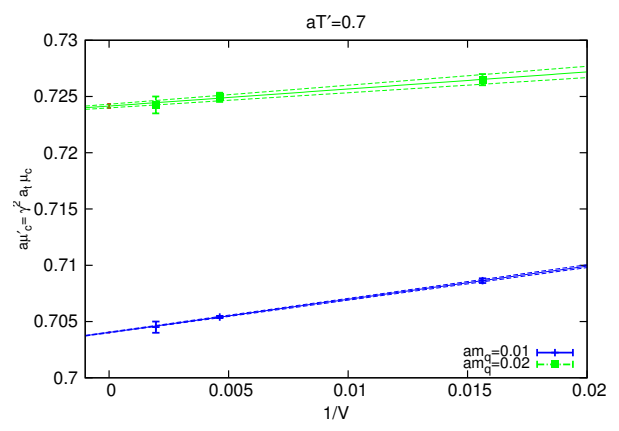

(b) Extrapolation to Thermodynamic limit

Figure 2: The left panel shows the Breit-Wigner fit to find the peak position. The right panel shows the extrapolation to the thermodynamic limit for various quark masses in fixed temperature $a T^{\prime}=0.7$.

way. First, we fit a peak of the susceptibility using the Breit-Wigner function with polynomial to find the peak position. The red lines in Fig. 2(a) are the peak position with fitting errors. After we get the $a_{t} \mu_{c}^{\prime}$, we do the extrapolation to the thermodynamic limit to eliminate the volume dependency. The results of extrapolations have very linear behavior with respect to $1 / V$ as shown in Fig. 2(b).
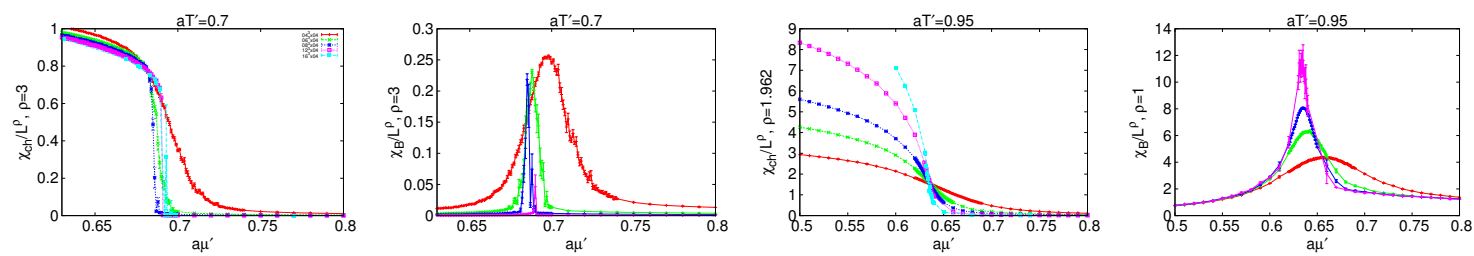

Figure 3: The chiral and baryon susceptibilities in the chiral limit. In the case of chiral susceptibility, we apply the finite size scaling with $\rho=1.962$ for the 2 nd order in the third panel, and $\rho=3$ for the 1 st order transition in the first panel. For the baryon susceptibility, we apply $\rho=1$ for crossover transition in the forth panel, and $\rho=3$ for the 1 st order transition in the second panel.

First, we address the results in the chiral limit comparing the 1st order lines from the nuclear and chiral transition. We plot the chiral and nuclear susceptibilities in Fig. 3. For the chiral transition at $a T^{\prime}=0.95$, we use the $O(2)$ exponents for 2 nd line. But the case of nuclear transition at $a T^{\prime}=0.95$, crossover scaling $\rho=1$ is applied. We apply finite size scaling with $\rho=3$ at $a T^{\prime}=0.7$ for both chiral and nuclear transitions because they are belonged in the temperature of 1 st order transition.

If we turn on the quark mass, the chiral susceptibility has a peak. We plot the chiral condensate and susceptibility for finite quark mass in Fig. 4(a). The baryon density and susceptibility for finite quark mass are plotted in Fig. 4(b). For the lower panels in Fig. 4(a) and Fig. 4(b), the $Z(2)$ exponents are applied. Then, the order of peak heights at $a T^{\prime}=0.725$ and those at $a T^{\prime}=0.75$ are opposite. Hence, we find that the temperature of the CEP is located between $0.725<a T^{\prime}<0.75$. The phase boundaries for finite quark masses are obtained from the peak analysis explained above. In the Table 1, our results of CEPs are agree with the previous Monte Carlo results for various 

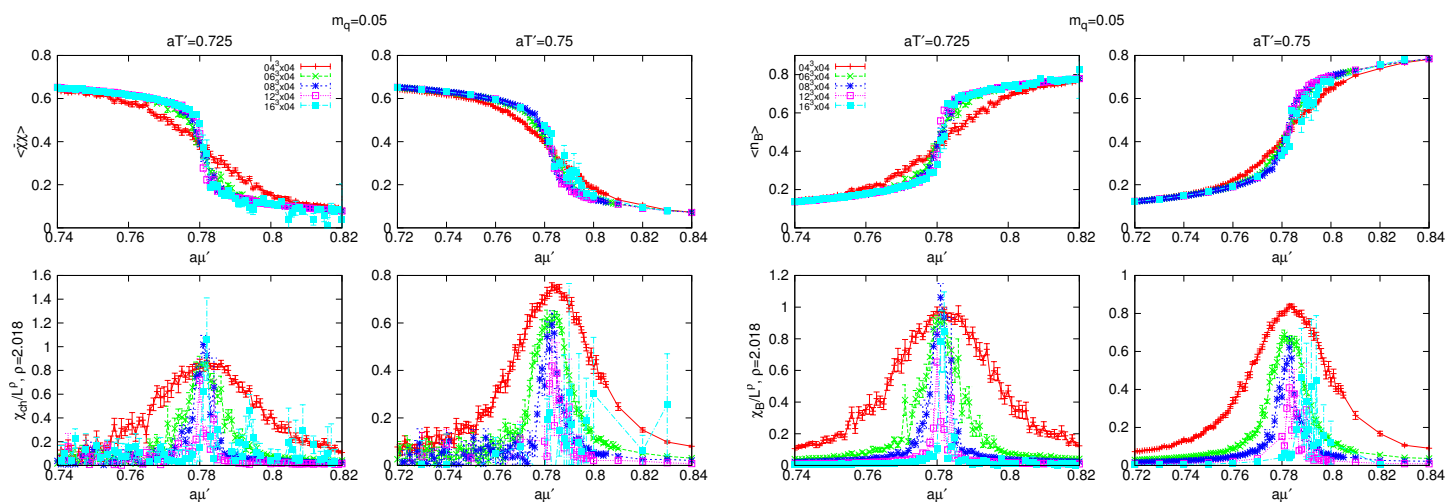

(a) Chiral transition

(b) Nuclear transition

Figure 4: The figure (a) shows the chiral condensate and the chiral susceptibility for finite quark mass $m_{q}=0.05$ near the critical end point temperature. The data is more noisy than those of nuclear transition due to the monomer fluctuations. The figure (b) shows the baryon density and the baryon susceptibility for finite quark mass $m_{q}=0.05$ near the critical end point temperature.

quark masses. We also compare the results to the Mean Field results in Table 1.

\begin{tabular}{lllll}
\hline \hline$a m_{q}$ & Previous MC $\left(a T_{E}^{\prime}, a \mu_{E}^{\prime}\right)$ & MeanField $\left(a T_{E}^{\prime}, a \mu_{E}^{\prime}\right)$ & $\operatorname{Ours}\left(a T_{E}^{\prime}, a \mu_{E}^{\prime}\right)$ & $\operatorname{Ours}\left(a T_{E}, a \mu_{E}\right)$ \\
\hline 0.00 & $0.94(7), 0.64_{-0.04}^{+0.02}$ & $0.866,0.577$ & $0.83(3), 0.6671(2)$ & $0.69(3), 0.5563(2)$ \\
0.01 & $0.77(3), 0.70(2)$ & $0.764,0.583$ & $0.78(3), 0.7005(5)$ & $0.66(3), 0.5906(4)$ \\
0.02 & N/A & N/A & $0.75(3), 0.7234(14)$ & $0.64(3), 0.6137(12)$ \\
0.05 & N/A & $0.690,0.617$ & $0.73(3), 0.7808(5)$ & $0.62(3), 0.6653(4)$ \\
0.10 & $0.69(1), 0.86(1)$ & $0.646,0.653$ & $0.70(3), 0.8606(10)$ & $0.60(3), 0.7386(9)$ \\
\hline \hline
\end{tabular}

Table 1: We compare our results of CEPs for various quark masses to the previous Monte Carlo results [3] and Mean Field results [8]. The forth column shows the results when we apply the correct anisotropy $\xi(\gamma)$.

\subsection{Phase diagram for finite quark masses}

Finally, we obtain the phase diagram for finite quark masses. We plot the phase diagrams applied $\xi^{\prime}(\gamma)=\gamma^{2}$ and $\xi(\gamma)$ in the first and second panels in Fig. 5. When we apply the $\xi^{\prime}(\gamma)$, they have back-bending in the low temperature region. This is because for $\gamma<1$ spatial dimers are favored, which results in an unphysical phase boundary. However, the back-bending disappears when the correct non-perturbative result $\xi(\gamma)$ is applied. The third panel shows the trajectory of CEPs and those of mean-field theory [8]. The $\mathrm{x}$-axis $\left(a m_{q}\right)^{2 / 5}$ in this panel is suggested by tricritical scaling. Due to the correct non-perturbative anisotropy $\xi(\gamma)$, the mismatch with mean-field theory has been enlarged. Just as $a T_{c}$ differs between Monte Carlo and mean-field theory, also the slope in $\left(a m_{q}\right)^{2 / 5}$ differs.

\section{Conclusion}

We obtain the phase boundary and critical end points for various quark masses using Monte Carlo simulation in the dual representation. We extend the 1st order phase boundary to the lower 

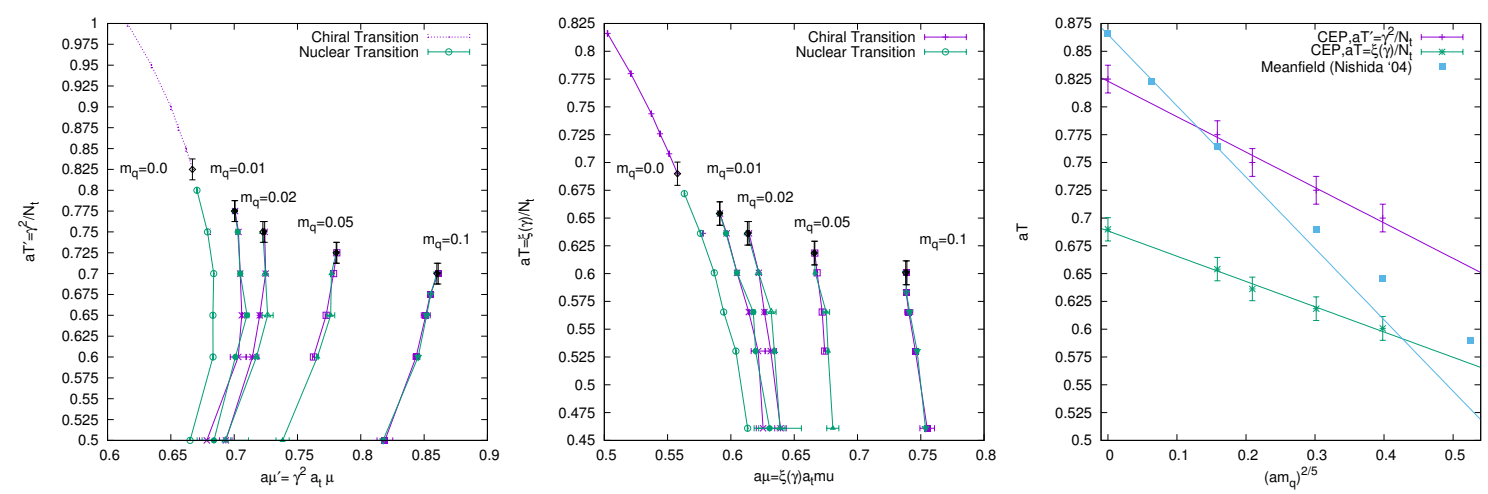

Figure 5: The first and second panels are the phase boundary of chiral and nuclear transition for finite quark masses. In the first panel, we apply the $\xi^{\prime}(\gamma)=\gamma^{2}$ for anisotropy. They have back-bending at the low temperature region. In the second panel, we apply $\xi(\gamma)$ obtained from non-perturbative calculation. After we apply the correct anisotropy, the back-bending has disappeared. The third panel is the trajectory of critical end points (from $\xi(\gamma)$ and $\xi^{\prime}(\gamma)$ ) and those of mean-field theory.

temperature than the previous Monte Carlo results. As expected, both the nuclear and chiral 1st order transitions are on top also for $m_{q}>0$. By applying the non-perturbative results for $\frac{a}{a_{t}} \equiv \xi(\gamma)$, we confirm the disappearance of back-bending for all quark masses.

\section{Acknowledgments}

Calculations leading to the results presented here were performed on resources provided by the Paderborn Center for Parallel Computing. We would like to thank Philippe de Forcrand and Helvio Vairinhos for helpful discussions.

\section{References}

[1] P. Rossi and U. Wolff, Lattice QCD With Fermions at Strong Coupling: A Dimer System, Nucl. Phys. B248 (1984) 105-122.

[2] D. H. Adams and S. Chandrasekharan, Chiral limit of strongly coupled lattice gauge theories, Nucl. Phys. B662 (2003) 220-246, [hep-lat/ 0303003 ].

[3] M. Fromm, Lattice QCD at string coupling: thermodynamics and nuclear physics, Thesis (2010).

[4] P. de Forcrand and M. Fromm, Nuclear Physics from lattice QCD at strong coupling, Phys. Rev. Lett. 104 (2010) 112005, [0907.1915].

[5] W. Unger and P. de Forcrand, Continuous Time Monte Carlo for Lattice QCD in the Strong Coupling Limit, PoS LATTICE2011 (2011) 218, [1111 . 1434].

[6] G. Faldt and B. Petersson, Strong Coupling Expansion of Lattice Gauge Theories at Finite Temperature, Nucl. Phys. B265 (1986) 197-222.

[7] P. de Forcrand, P. Romatschke, W. Unger, and H. Vairinhos, Thermodynamics of strongly-coupled lattice QCD in the chiral limit, PoS LATTICE2016 (2016) 086.

[8] Y. Nishida, Phase structures of strong coupling lattice QCD with finite baryon and isospin density, Phys. Rev. D69 (2004) 094501, [hep-ph/ 0312371$].$ 